\title{
TAMAÑO POBLACIONAL Y CARACTERÍSTICAS DEL HÁBITAT DE MAMMILLARIA ERIACANTHA, UNA CACTÁCEA ENDÉMICA Del Centro de Veracruz, México
}

\author{
Susana Valencia-Díaz ${ }^{1,3}$, Alejandro Flores-Palacios ${ }^{1}$ y Gonzalo Castillo-Campos² \\ ${ }^{1}$ Centro de Investigación en Biodiversidad y Conservación, Universidad Autónoma del Estado de Morelos. \\ ${ }^{2}$ Red de Biodiversidad y Sistemática, Instituto de Ecología, A.C. \\ ${ }^{3}$ Autor para la correspondencia: susana.valencia@uaem.mx
}

\begin{abstract}
Resumen: La fragmentación del hábitat disminuye las poblaciones y puede ocasionar la desaparición de especies con distribución restringida. Al tener un alto grado de endemismo, muchas cactáceas son vulnerables a la fragmentación. Mammillaria eriacantha, una cactácea endémica del centro de Veracruz, habita 22 fragmentos de vegetación aislados entre sí y rodeados por cultivos. En este trabajo el objetivo fue estimar el tamaño poblacional de $M$. eriacantha y describir algunas características poblacionales y de su hábitat considerando a la fragmentación como variable en el diseño de muestreo. Con base en un muestreo por conglomerados en dos etapas, se eligieron aleatoriamente seis fragmentos de vegetación. Al interior de cada fragmento, se eligieron al azar diez parcelas de $5 \times 5 \mathrm{~m}$, donde se registró la abundancia de $M$. eriacantha, plantas en floración, la altura de cada individuo y la distancia a la $M$. eriacantha más cercana. En cada parcela, se registró el porcentaje de rocosidad y de cobertura de hierbas y del dosel. Se describió la estructura de tamaños de $M$. eriacantha, se determinó su densidad, tamaño poblacional, número de adultos y su patrón de distribución espacial. Se correlacionaron las variables poblacionales con las de hábitat. El tamaño poblacional estimado de $M$. eriacantha para el área total $\left(9.53 \mathrm{~km}^{2}\right)$ fue de 1,081,125.7 plantas. Mammillaria eriacantha se distribuyó homogéneamente. Las variables de hábitat y los atributos poblacionales de M. eriacantha no correlacionaron significativamente. El tamaño poblacional de M. eriacantha es grande; pero, la transformación de su hábitat y el saqueo podrían alterar su estructura poblacional y abundancia. Es importante establecer bases para el manejo y conservación de esta especie.
\end{abstract}

Palabras clave: bosque tropical caducifolio, estructura poblacional, fragmentación, México, tamaño poblacional, Veracruz.

\begin{abstract}
Habitat fragmentation reduces populations and can cause disappearance in species of restricted distribution. High degrees of endemism make many cacti species vulnerable to fragmentation Mammillaria eriacantha, a cactus endemic to central Veracruz, is restricted mainly to 22 isolated vegetation fragments each surrounded by cultivated land. The objective of this study was to estimate the population size of $M$. eriacantha and describe certain characteristics of the population and its habitat, considering fragmentation as a variable in the sampling design. Based on two-stage cluster sampling, six fragments of vegetation were randomly chosen. Within each fragment, ten plots of $5 \times 5 \mathrm{~m}$ were randomly selected and the abundance of $M$. eriacantha within each plot was recorded, along with number of flowering adults, height of each individual and distance to the closest neighbouring $M$. eriacantha. In each plot, percentage of rockiness and coverage of grasses and canopy was recorded. Size structure of $M$. eriacantha was described, as well as its density, population size, number of adults, and spatial distribution pattern. Population variables were correlated with those of habitat. The estimated $M$. eriacantha population size for the total fragment area $\left(9.53 \mathrm{~km}^{2}\right)$ was $1,081,125.7$ plants. Mammillaria eriacantha distribution was homogeneous, and no habitat variable was found to correlate with the measured $M$. eriacantha population characteristics. Notwithstanding the considerable size of the $M$. eriacantha population, the ongoing transformation of its habitat by exploitation and modification for agriculture could modify its population structure and abundance, so the establishment of sound management and conservation of this species is an important issue.
\end{abstract}

Key words: fragmentation, Mexico, population size; population structure, tropical dry forest, Veracruz.

$\mathbf{E}_{\mathrm{p}}^{1}$ tamaño poblacional tiene una relación directa con la permanencia de las poblaciones y en consecuencia con la probabilidad de extinción de las especies (Reed, 2004), ya que entre más pequeña sea una población menor será su diversidad genética, estará sujeta a azar demográfico y au- mentará su probabilidad de extinción (Karron, 1997; Morgan, 1999; Vergeer et al., 2003).

La deforestación afecta el tamaño y distribución de las poblaciones al reducir y fragmentar el hábitat (Sih et al., 2000; Jacquemyn et al., 2003). La fragmentación ocurre 
cuando un hábitat es transformado y sólo quedan remanentes o "islas de hábitat" aislados entre sí y rodeados por una matriz diferente a la original (Wilcove et al., 1986; Guevara et al., 2004). Son muchas las especies de plantas amenazadas o en peligro de extinción como consecuencia de este fenómeno; pero en especies endémicas y de distribución restringida, esto se magnifica, ya que cualquier factor que disminuya su tamaño poblacional las acerca a la extinción (Frankham, 1998; Morgan, 1999; Keith, 2000).

Aunque la investigación del tamaño poblacional y de otras características poblacionales (Menges y Dolan, 1998; Keith, 2000) de especies de plantas en ambientes fragmentados no es nueva; hasta donde sabemos, no existen estudios de estimación estadística del tamaño poblacional de plantas y que involucren en el diseño de muestreo a la fragmentación como variable. Si se considera lo anterior, se conocerá donde ocurre la mayor variabilidad en la estimación del tamaño poblacional, entre fragmentos de vegetación o en su interior. Esto permitirá ponderar los costos de la estimación del tamaño poblacional, decidiendo invertir entre muestreos intensivos dentro de un fragmento de vegetación o extensivos en la mayor cantidad de fragmentos. Lo anterior puede contribuir al monitoreo y a la elaboración de planes de conservación de plantas en ambientes fragmentados.

La familia Cactaceae está entre las familias con más especies amenazadas internacionalmente. En México 35\% de las cactáceas se encuentran amenazadas (Bárcenas-Luna, 2003) y además existe un alto endemismo a nivel de especie $(78 \%)$ y de género $(73 \%)$ (Hernández y Godínez, 1994). El género con más especies protegidas es Mammillaria (39.4\%; SEMARNAT, 2010). Mammillaria eriacantha Link \& Otto ex Pfeiff., una especie endémica del centro de Veracruz (Bravo-Hollis y Sánchez-Mejorada, 1991) habita sólo 22 fragmentos de vegetación (Figura 1) como consecuencia de la reducción de su hábitat (Castillo-Campos, 2003). Mammillaria eriacantha es una especie que ha sido categorizada como vulnerable por el CITES (Hunt, 1992) y

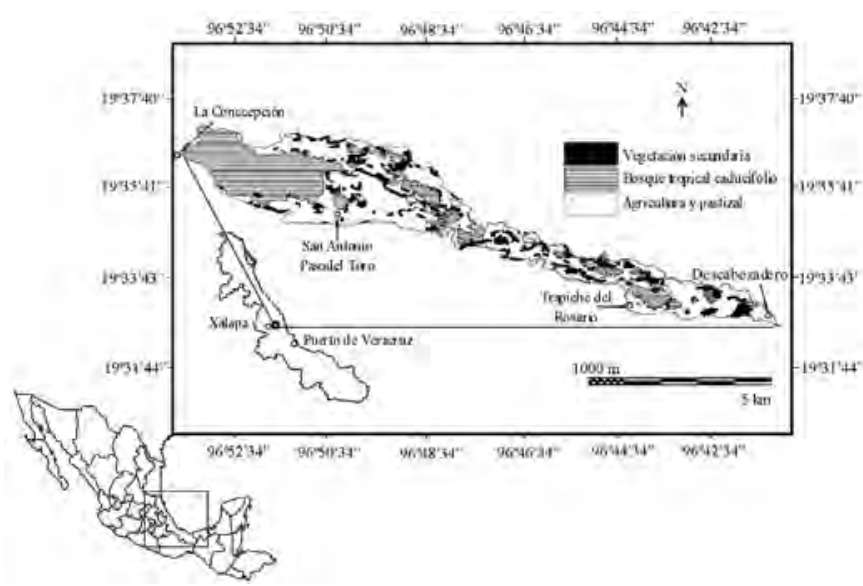

Figura 1. Fragmentos de bosque tropical caducifolio en el centro de Veracruz y donde se distribuye Mammillaria eriacantha. propuesta como especie en peligro de extinción (Vovides et al., 1997). No obstante; se extraen ejemplares de su hábitat para su comercio ilegal (Flores-Palacios y Valencia-Díaz, 2007) y hasta el momento no existen estudios poblacionales sobre la misma. Por lo que el objetivo de este trabajo fue estimar el tamaño poblacional de $M$. eriacantha, así como describir algunas características poblacionales y de su hábitat considerando a la fragmentación como variable en el diseño de muestreo.

\section{Materiales y métodos}

Especie y zona de estudio. Mammillaria eriacantha es una cactácea cilíndrica y alargada; posee tubérculos verdes, próximos entre sí. Las areolas son ovales, con dos espinas centrales, de 8 a $10 \mathrm{~mm}$ de longitud, las espinas superiores son más cortas. Tiene flores amarillo-verdosas laterales de $15 \mathrm{~mm}$ de longitud y diámetro que emergen en la mitad del tallo. El fruto es blanco verdoso, anaranjado o rojizo, claviforme, de $10 \mathrm{~mm}$ de longitud (Bravo-Hollis y Sánchez-Mejorada, 1991). Florece a finales de invierno (febrero-marzo) y fructifica en primavera (marzo-abril, obs. pers.).

La zona de estudio $\left(19^{\circ} 33^{\prime}\right.$ y $19^{\circ} 36^{\prime} \mathrm{N}$ y $96^{\circ} 50^{\prime}$ y $96^{\circ} 55^{\prime}$ O) incluye diversas localidades de los municipios de Naolinco, Alto Lucero y Actopan en el estado de Veracruz. La temperatura media anual es de $24.7{ }^{\circ} \mathrm{C}$ y la precipitación anual es de 899 mm (García, 1988). La vegetación característica de esta zona es bosque tropical caducifolio, que se desarrolla sobre un derrame de lava volcánica de aproximadamente 10,000 años de antigüedad (Castillo-Campos, 2003; CastilloCampos et al., 2008). Como consecuencia de las actividades agrícolas y ganaderas, este tipo de vegetación en la región de estudio se ha fragmentado y reducido a 22 fragmentos de vegetación original (1,387 ha), con tamaños que van desde menos de 1 ha hasta 953 ha (Castillo-Campos, 2003).

En el bosque tropical caducifolio de la zona de estudio se han registrado 105 familias, 390 géneros y 682 especies de plantas, las familias más numerosas en especies son Asteraceae, Euphorbiaceae y Fabaceae (Castillo-Campos et al., 2008). Las familias Bromeliaceae, Cactaceae, Euphorbiaceae y Orchidaceae tienen el mayor número de especies suculentas en el sitio de estudio (Castillo-Campos, 2003). También se tiene registrado un alto grado de endemismo, ya que $7 \%$ del total de especies son endémicas de Megaméxico 3 y de éstas $15.2 \%$ son endémicas del Centro de Veracruz (Castillo-Campos, 2003).

Muestreo. Con la finalidad de considerar la fragmentación del hábitat como variable en la estimación del tamaño poblacional de Mammillaria eriacantha, se empleó el muestreo por conglomerados en dos etapas (Schaeffer et al., 1986). Este método se emplea cuando existe un gran número de conglomerados en la población (primera etapa). Además cada conglomerado se subdivide a su vez en un gran número 
de unidades de muestreo (p. ej. transectos o parcelas), por lo que se elige al interior de cada conglomerado una muestra de ellos (segunda etapa; Schaeffer et al., 1986). El muestreo se realizó de mayo de 2002 a febrero de 2003.

En este estudio cada conglomerado fue equivalente a un fragmento de vegetación $(\mathrm{N}=22)$. En la primera etapa del muestreo se eligió una muestra aleatoria de seis fragmentos de vegetación. Posteriormente, en la segunda etapa se eligieron al azar diez parcelas de $25 \mathrm{~m}^{2}(5 \times 5 \mathrm{~m})$ al interior de cada fragmento. Para esto se cuadricularon los mapas de los fragmentos de vegetación y con base en las coordenadas se ubicaron en campo las parcelas con un receptor del sistema de posicionamiento geográfico. Los mapas utilizados fueron elaborados con ortofotos del año 1995, y analizados con ArcView 3.2a para Windows. Para la delimitación de las unidades de vegetación y uso del suelo, se utilizó como criterio principal el tono, la textura y el tipo de grano de las ortofotos. Posteriormente se llevo a cabo una verificación de campo y muestreos florísticos para la caracterización de las comunidades vegetales (Castillo-Campos, 2003).

Este tipo de muestreo involucró, entonces, la selección de dos tamaños de muestra, la muestra de fragmentos de vegetación ( $n$ ) y la muestra de parcelas dentro de cada fragmento elegido $(m)$. Para obtener $n$ y $m$ adecuadas se deben de conocer las varianzas de la abundancia de Mammillaria ericantha que existen dentro de los fragmentos de vegetación $\left(\mathrm{s}_{\mathrm{w}}^{2}\right)$ y entre ellos $\left(\mathrm{s}_{\mathrm{b}}^{2}\right)$. El tamaño de muestra $\mathrm{m}$ se incrementa conforme aumenta $\mathrm{s}^{2}{ }_{\mathrm{w}}$ y disminuye cuando $\mathrm{s}_{\mathrm{b}}{ }_{\mathrm{b}}$ aumenta. Otras variables que se consideraron fueron el costo de muestreo $(C)$ dentro y entre los fragmentos (ya que $m$ está en función de éste) y una varianza fijada por el investigador (VFI), que representa un porcentaje de la varianza total que se está dispuesto a tolerar en el muestreo, en este caso $5 \%$. $C, n$ y $m$ se obtuvieron de acuerdo con las siguientes fórmulas (Schaeffer et al., 1986):

$$
C=\frac{c_{\text {entrefragmentes }}}{c_{\text {dentrofragmentos }}}
$$

donde $C_{\text {entrefragmentos }}$; considera el costo del transporte, alimentación, un ayudante y el tiempo necesario para localizar, desplazarse entre los fragmentos de vegetación y/o gestionar el acceso con los propietarios. $\mathrm{El} C_{\text {dentrodefragmentos }}$; es el costo por muestrear al interior de cada fragmento. Este costo fue más bajo, pues era relativamente más fácil desplazarse y muestrear al interior de los fragmentos una vez que se tenía acceso a ellos. Por otro lado, el costo por localizar los fragmentos fue más alto porque los fragmentos debían ser rodeados hasta tener un acceso entre la matriz de cañaverales y propiedades privadas.

$$
\mathrm{m}=\sqrt{\frac{s_{w}^{2}}{s_{b}^{2}} \cdot C} \quad, \mathrm{n}=\frac{s_{b}^{2}+\frac{s_{w w}^{2}}{m}}{V F I}
$$

Con la finalidad de estimar el tamaño poblacional, la estructura de tamaños, la distribución espacial y las características del hábitat de Mammillaria eriacantha; en cada parcela se registró el número de individuos de $M$. eriacantha y la altura de cada uno de ellos y se midió, dentro de la misma especie, la distancia al vecino más cercano (VMC). Debido a que $M$. eriacantha crece en zonas rocosas en el sotobosque y a que su presencia pudiera estar relacionada con la cobertura vegetal (Castillo-Campos, 2003), en cada parcela se registraron los porcentajes de rocosidad y de cobertura de hierbas y del dosel, los cuales se calcularon de acuerdo con el índice de cobertura-abundancia de Braun-Blanquet (Mueller-Dombois y Ellenberg, 1974). La cobertura del dosel se estimó utilizando un densiómetro (Forestry Supplier, Bartlesville OK, Modelo-C), el cual mide la sombra que proyecta el dosel en un espejo cóncavo.

Estructura de tamaños de Mammillaria eriacantha. La estructura poblacional de $M$. eriacantha en el área total muestreada y por fragmento de vegetación, se describió en ocho categorías de altura a intervalos de $5 \mathrm{~cm}$ cada una $(<5 \mathrm{~cm}$, 5.1-10 cm, 10.1-15 cm, 15.1-20 cm, 20.1-25 cm, 25.1-30 $\mathrm{cm}, 30.1-35 \mathrm{~cm} \mathrm{y}>35 \mathrm{~cm}$ ). Con base en estas categorías se realizaron histogramas de frecuencias. Para probar si las poblaciones de $M$. eriacantha tienen una estructura de tamaños similar entre fragmentos, se realizó un análisis de cuadro de contingencia, acompañado de una prueba de residuos estandarizados de Haberman (Haberman, 1973). En caso de que al menos un fragmento tuviera una población de M. eriacantha diferente de las demás en cuanto a la abundancia de una categoría de altura, los residuos tomarán entonces valores mayores de |2|, y el sentido de la diferencia lo indicará el signo del residuo (Haberman, 1973).

Estimación del tamaño poblacional y del número de adultos de Mammillaria eriacantha. Una vez contabilizados los individuos de $M$. eriacantha, el tamaño poblacional $(\hat{\boldsymbol{M}})$ de esta especie se estimó con la fórmula (Schaeffer et al., 1986):

$$
\hat{M}=\hat{\lambda} A
$$

donde $A$ es la suma del área de todos los fragmentos de vegetación y $\hat{\lambda}$ es el estimador de la densidad que se obtiene con la fórmula:

$$
\hat{\lambda}=\frac{\bar{m}}{a}
$$

donde $a$, es el total del área muestreada en los seis fragmentos; es el promedio de $\bar{m}$ de todas las parcelas muestreadas; $m$, es el número de individuos de $M$. eriacantha en cada parcela muestreada.

Con la finalidad de estimar el número total de adultos (individuos en floración), de una submuestra de 300 individuos elegidos al azar, se registraron aquellos con flores y de acuerdo con su altura se determinó la categoría de tamaños a la que pertenecían. Se realizó un análisis de regresión sim- 
ple (Zar, 2010) para determinar si la floración de individuos de Mammillaria eriacantha estaba en función de su altura, y por lo tanto establecer un límite inferior de tamaños a partir del cual los individuos de esta especie serían considerados adultos.

Patrón de distribución espacial de Mammillaria eriacantha. El conocimiento de los patrones de distribución de las especies vegetales es importante pues permite diseñar planes de conservación (Hou et al., 2010), por lo que para conocer si la distribución espacial de $M$. eriacantha era agregada, uniforme o al azar, se calculó el índice de distribución espacial (R, Clark y Evans, 1954), por medio de la fórmula:

$$
\begin{gathered}
R=\frac{r A}{r E} \\
\text { donde: } \quad r A=\frac{\sum r}{N}, r E=\frac{1}{2} \sqrt{\rho}
\end{gathered}
$$

$r$ es la distancia de un individuo a su VMC, $N$ es el número de mediciones de distancia, $p$ es la densidad de la distribución observada (número de individuos/unidad de área). De acuerdo con lo anterior, si $R=0$, la distribución es agregada; si $R=1$, la distribución azarosa; y si $R>1$, la distribución es homogénea (Clark y Evans, 1954).

El promedio del $R \pm$ D.E. y los intervalos de confianza (I.C.) se calcularon para todos los fragmentos de vegetación en conjunto y para cada fragmento en particular. Si el I.C. incluyó al 0 , se consideró una población agregada; si $0 \geq$ I.C $<1$, entonces $R$ indicó una distribución al azar; y si el I.C. incluyó al 1, entonces se consideró que la distribución era homogénea.

Relación entre características del hábitat y demográficas de Mammillaria eriacantha. Se realizaron correlaciones simples (Zar, 2010) para asociar las características poblacionales de $M$. eriacantha con las características de su hábitat. Las características poblacionales fueron densidad y distribución de estructura de tamaños (representada por su valor de asimetría) y las características del hábitat fueron rocosidad y coberturas de hierbas y del dosel. El valor de la asimetría representa como es la distribución de los individuos de $M$. eriacantha en las categorías de altura. La asimetría es positiva $>0$ cuando hay más individuos en las categorías de altura de menor tamaño, negativa $<0$ cuando hay más individuos en las categorías de altura de mayor tamaño y simétrica $=0$ cuando los individuos se distribuyen uniformemente. Todos los análisis se realizaron con el programa STATISTICA (Stat Soft, Tulsa, OK, USA).

\section{Resultados}

Muestreo. Con valores de 2,536.2 y 2,562.90 para $s^{2}{ }_{w}$ y $s_{b}^{2}$, respectivamente; y con un costo de 80 (lo cual significa que
Cuadro 1. Abundancia de individuos de Mammillaria eriacantha, media y varianza en diez parcelas muestreadas en cada uno de los seis fragmentos muestreados de bosque tropical caducifolio del centro de Veracruz. Se muestra también el área de cada fragmento.

\begin{tabular}{ccccc}
\hline Fragmentos & Área $\left(\mathrm{Km}^{2}\right)$ & $\begin{array}{c}\text { No. } \\
\text { individuos }\end{array}$ & Media & Varianza \\
\hline 1 & 0.39 & 216 & 21.6 & 155.1 \\
2 & 0.21 & 210 & 21.0 & 158.4 \\
3 & 0.31 & 144 & 14.4 & 127.8 \\
4 & 0.02 & 162 & 16.2 & 85.95 \\
5 & 0.05 & 176 & 17.6 & 138.5 \\
6 & 0.90 & 112 & 11.2 & 45.7 \\
\hline
\end{tabular}

es 80 veces más costoso desplazarse entre fragmentos de vegetación que muestrear en su interior), se estimó un tamaño de muestra dentro de los fragmentos de vegetación (m) de 8.89 lo cual se redondeó a nueve parcelas como un tamaño de muestra suficiente para el muestreo dentro de los fragmentos. Con una varianza fijada por el investigador del $5 \%$ se obtuvo un $\mathrm{n}=3.74$, lo cual se redondeó a cuatro fragmentos para la estimación del tamaño poblacional total en la zona de estudio.

En el área total muestreada $\left(\mathrm{n}=60\right.$ parcelas, $\left.1,500 \mathrm{~m}^{2}\right)$ se registraron 1,020 individuos de Mammillaria eriacantha (Cuadro 1). El promedio ( \pm D. E.) de plantas por fragmento fue de $170.16 \pm 36.18$ y por parcela fue de $12.49 \pm 9.62$. El $75 \%$ de las parcelas tuvieron de 0-18 plantas.

Estructura de tamaños de Mammillaria eriacantha. La altura promedio de los individuos de $M$. eriacantha fue de $12.15 \mathrm{~cm} \pm 2.93 \mathrm{~cm}$. La mayor parte de los individuos estuvieron en las primeras categorías $(<10 \mathrm{~cm})$ y su abundancia disminuyó en las categorías de mayor altura (Figura 2). Se encontraron diferencias en la distribución de tamaños de $M$. eriacantha entre fragmentos $\left(\chi^{2}=81.2\right.$, g.1. $\left.=35, P<0.05\right)$.

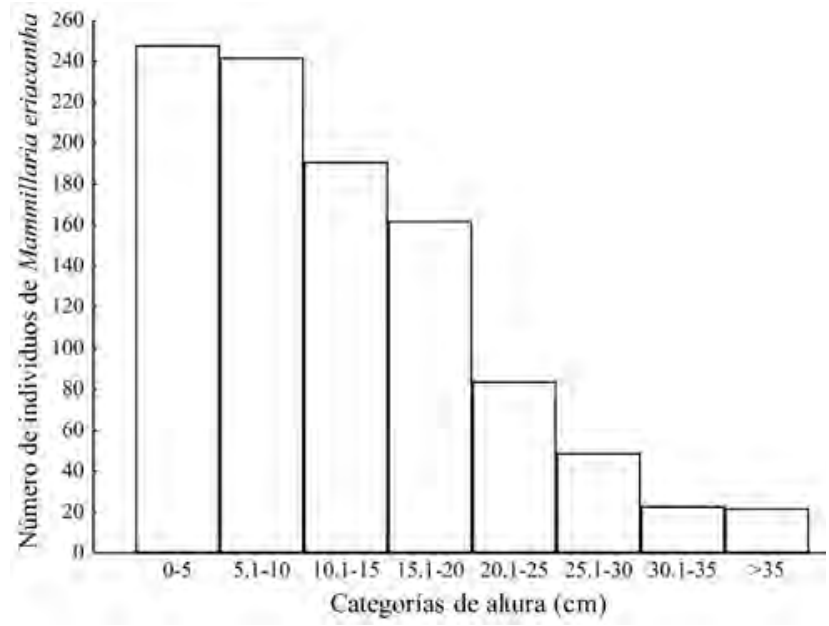

Figura 2. Estructura de tamaños (altura) de la población total muestreada de Mammillaria eriacantha en la zona de estudio. 

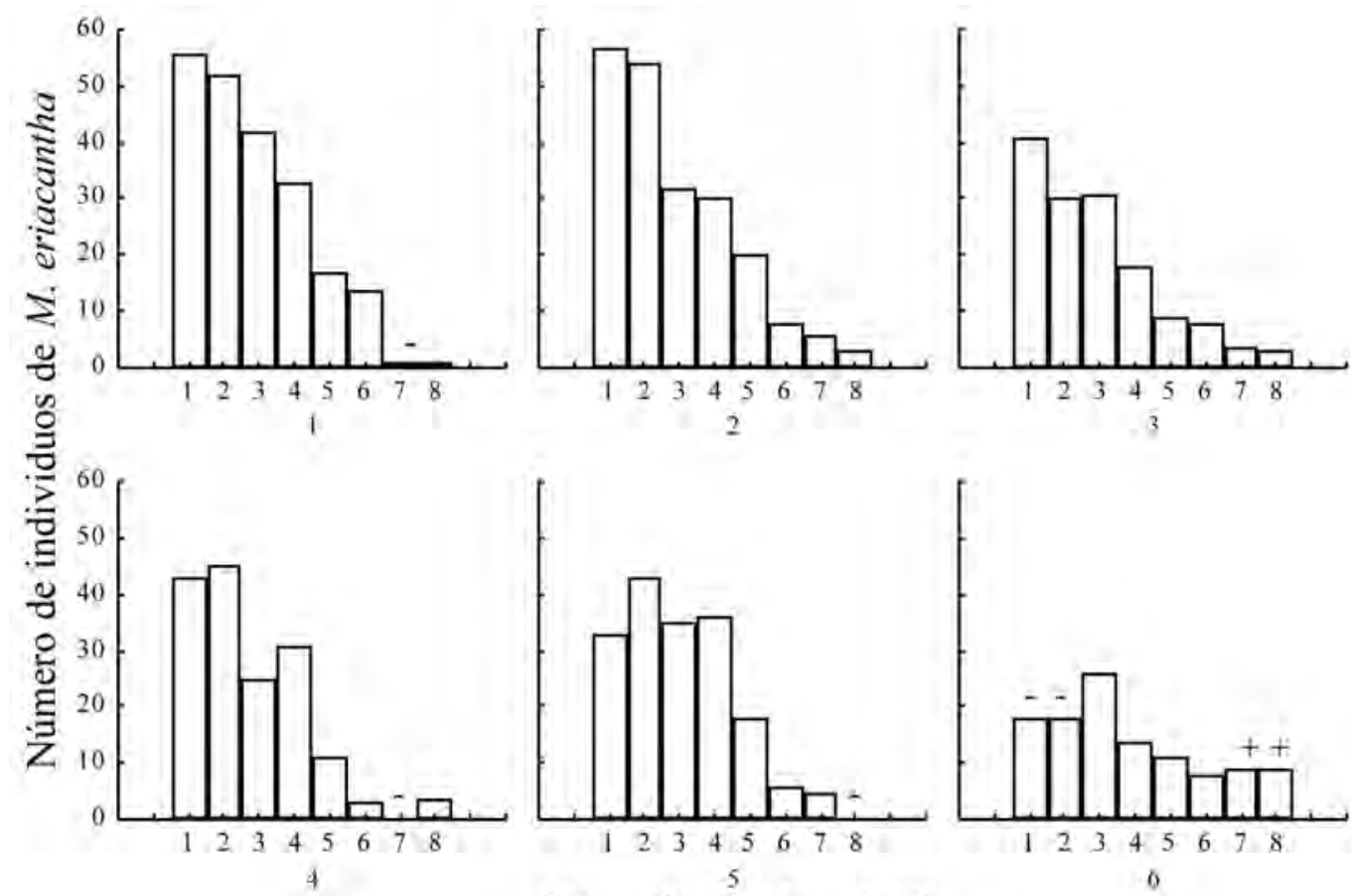

Categorías de altura $(\mathrm{cm})$

Figura 3. Estructura de tamaños (altura), de las poblaciones de Mammillaria eriacantha en cada fragmento. Las categorías son: $1(<5$ $\mathrm{cm}), 2(5.1-10 \mathrm{~cm}), 3(10.1-15 \mathrm{~cm}), 4(15.1-20 \mathrm{~cm}), 5(20.1-25 \mathrm{~cm}), 6(25.1-30 \mathrm{~cm}), 7(30.1-35 \mathrm{~cm})$ y $8(>35 \mathrm{~cm})$. Los signos - arriba de las columnas representan un menor número de individuos de $M$. eriacantha de lo que se esperaría por azar en esa categoría; y los signos + representan un mayor número de individuos de $M$. ericantha de lo que se esperaría por azar en esa categoría.

En los fragmentos uno, cuatro y cinco hubo un déficit de individuos adultos de las categorías siete y ocho; en el fragmento seis hubo un déficit de individuos en las primeras dos categorías de tamaño y exceso de las dos últimas, mientras que en los fragmentos dos y tres los individuos se distribuyeron en su mayoría en las categorías de menor tamaño y disminuyeron paulatinamente su frecuencia hacia las categorías de mayor altura (Figura 3).

Estimación del tamaño poblacional de Mammillaria eriacantha. Para estimar $M$, se emplearon los siguientes valores:

$\mathrm{n}=6$ fragmentos $, \sum_{i=1}^{n} m=1,020, \mathrm{a}=0.0015 \mathrm{~km}^{2}, \bar{m}=170.16$, $\lambda=113,444.5$ y $\tilde{V}(\dot{M})=12,604,940.7$. El tamaño poblacional estimado de Mammillaria eriacantha para el área total cubierta por los 22 fragmentos $\left(9.53 \mathrm{~km}^{2}\right)$ fue de 1,081,125.7 $\pm 3,550.3$ ( $\dot{M}$ limite error de estimación).

De 300 individuos de Mammillaria eriacantha muestreados al azar, 54 (18\%) tuvieron flor. La frecuencia de plantas en floración se incrementó conforme a la altura de los individuos (Figura 4). A partir de los $9 \mathrm{~cm}$ de altura, se encontraron plantas floreciendo. No se registraron plantas con flores mayores que $35 \mathrm{~cm}$ porque sólo hubo dos plantas con altura mayor que $35 \mathrm{~cm}$.

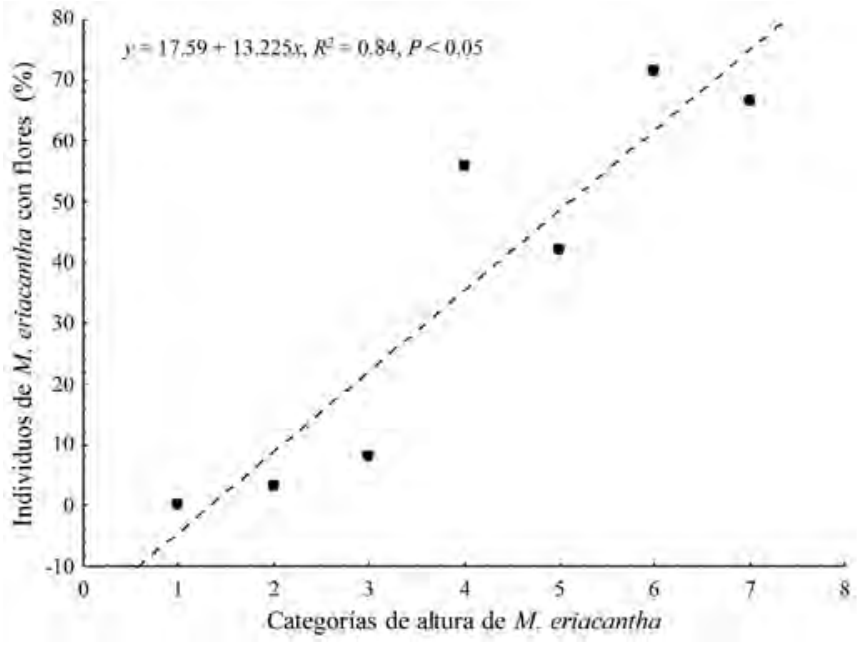

Figura 4. Siete categorías de altura de plantas de Mammillaria eriacantha y su relación con la floración. Las categorías son: 1 (< $5 \mathrm{~cm}), 2$ (5.1 - $10 \mathrm{~cm}), 3(10.1-15 \mathrm{~cm}), 4(15.1-20 \mathrm{~cm}), 5(20.1$ - $25 \mathrm{~cm}), 6(25.1-30 \mathrm{~cm}), 7(30.1-35 \mathrm{~cm})$. La línea interrumpida indica un ajuste lineal.

Patrón espacial de Mammillaria eriacantha. La distancia promedio al VMC fue de $44.99 \mathrm{~cm} \pm 54.98 \mathrm{~cm}$. El $75 \%$ de las plantas estuvieron a una distancia menor o igual a 65 $\mathrm{cm}$. Los datos de la distancia al VMC muestran que los in- 
Cuadro 2. Índice de distribución espacial de Mammillaria eriacantha con sus intervalos de confianza al 95\% (I.C.), para cada fragmento en particular y en su totalidad.

\begin{tabular}{ccc}
\hline $\begin{array}{c}\text { Fragmento de } \\
\text { vegetación }\end{array}$ & $R(x \pm D . E)$. & I.C. \\
\hline 1 & $1.021 \pm 0.613$ & $0.581-1.460$ \\
2 & $1.354 \pm 0.936$ & $0.684-2.025$ \\
3 & $3.493 \pm 4.964$ & $0.058-7.045$ \\
4 & $1.634 \pm 0.924$ & $0.970-2.291$ \\
5 & $2.751 \pm 3.255$ & $0.422-5.080$ \\
6 & $2.060 \pm 1.237$ & $1.100-3.012$ \\
General & $2.052 \pm 2.590$ & $1.370-2.720$ \\
\hline
\end{tabular}

dividuos de $M$. eriacantha tienden a presentarse a distancias cortas entre ellas. De acuerdo con el índice de patrón espacial $(R)$, por cada sitio y en general, la distribución espacial de $M$. eriacantha fue homogénea (Cuadro 2).

Relación entre características demográficas y del hábitat de Mammillaria eriacantha. El promedio de la rocosidad fue de $82.17 \% \pm 25.5 \%$, de la cobertura de hierbas fue de $51.08 \% \pm$ $20.02 \%$ y de la cobertura del dosel de $38.49 \% \pm 26.68 \%$.

La densidad promedio de Mammilaria eriacantha fue de $0.68 \pm 0.44$ individuos $/ \mathrm{m}^{2}$. Hubo desde parcelas donde no hubo ninguna planta, hasta parcelas donde se encontraron 1.8 individuos $/ \mathrm{m}^{2}$, pero la mayoría de las parcelas $(75 \%)$ tuvieron una densidad de 0.96 individuos $/ \mathrm{m}^{2}$; es decir, casi un individuo por metro cuadrado.

No se observó correlación alguna entre las variables poblacionales y las características del hábitat, aunque sí entre las características de hábitat consideradas $(\mathrm{r}=-0.6, P<$ 0.05; para rocosidad con la cobertura del dosel).

\section{Discusión}

Se considera que el tamaño de muestra de este estudio es representativa debido a que se muestreó un área más grande que la sugerida por las varianzas dentro y entre los fragmentos. Se muestrearon seis fragmentos de vegetación y con cuatro hubieran sido suficiente. Se muestrearon diez parcelas dentro de ellos pero con nueve ya había representatividad.

En estudios poblacionales de plantas en ambientes fragmentados, es importante decidir entre muestrear al interior de pocos fragmentos de vegetación o extensivamente entre más fragmentos. Las varianzas obtenidas en cada etapa del muestreo permiten decidir entre estas alternativas, ponderando los costos de muestreo. De acuerdo con los resultados obtenidos; en estudios de monitoreo y para conocer la estructura y densidad de Mammillaria eriacantha es mejor usar pocas parcelas dentro de pocos fragmentos; aún si la relación de costos es diez veces mayor entre fragmentos que dentro de ellos. Esto debido a que las varianzas en am- bas etapas fueron parecidas $\left(\mathrm{s}_{\mathrm{w}}^{2} / \mathrm{s}^{2}{ }_{\mathrm{b}}=0.99\right)$, pero podría no haber sido el caso, especialmente si la heterogeneidad de hábitat incrementara la varianza del muestreo. Aún con la recomendación previa, nuestros datos también señalan fragmentos donde el monitoreo debe realizarse para asegurar la permanencia de poblaciones con estructuras donde hay déficit de adultos o juveniles.

Existe evidencia de que las cactáceas tienen un patrón de distribución agregado (Godínez-Álvarez et al., 2003), probablemente debido al nodrizaje que les proporcionan algunas especies de plantas (Valiente-Banuet et al.,1991; Larrea-Alcázar et al., 2005), pero esto ha sido documentado en cactáceas de ambientes secos. Si bien el bosque tropical caducifolio de la zona de estudio es una ambiente seco la mayor parte del año, es probable que su clima no sea tan extremoso como para evidenciar un patrón de distribución agregado de Mammmillaria eriacantha. Por otro lado, es probable que el patrón de distribución de $M$. eriacantha obedezca a su síndrome de dispersión ornitocorio con semillas contenidas en frutos rojos (obs. pers.), ya que se ha documentado que la distribución de las especies vegetales es acorde con síndrome de dispersión (Seidler y Plotkin, 2006). Las especies de plantas cuyas semillas son dispersadas por animales, tienden a tener distribuciones menos agregadas que aquellas dispersadas por otros vectores como viento o gravedad (Seidler y Plotkin, 2006). Pero en el caso específico de $M$. eriacantha esto debe ser estudiado.

Se conoce que las plantas pequeñas tienen una mayor tasa de mortalidad (Silvertown y Lovett-Doust, 1993), ya que son más susceptibles a los enemigos naturales, a los disturbios naturales y a las perturbaciones antropogénicas (Gilbert, 2002; Bell et al., 2006; Drezner y Lazarous, 2008). En este estudio, Mammillaria eriacantha tuvo una alta proporción de plantas pequeñas (altura $<10 \mathrm{~cm}$ ), es probable que atributos como la germinación y/o reclutamiento de plántulas no limiten el tamaño poblacional de esta especie, pero hacen falta estudios específicos para probarlo. Así mismo, se ha sugerido que la mortalidad de plantas disminuye conforme se incrementa su tamaño (Godínez-Álvarez et al., 2003), en este estudio se observó que conforme las plantas eran más grandes (altura $>10 \mathrm{~cm}$ ) su abundancia disminuía, esto no necesariamente significa que mueren más adultos, pero es probable que su extracción se concentre en ejemplares mayores a $10 \mathrm{~cm}$. Las plantas mayores a $10 \mathrm{~cm}$ son más vistosas por la presencia de flores y además su comercialización se facilita (Flores-Palacios y Valencia-Díaz, 2007).

La deforestación ha fragmentado el hábitat ocasionando que las poblaciones queden aisladas y a su vez se encuentren separadas de otras poblaciones de su misma especie (Harrison, 1991; Hanski, 1998). La fragmentación del hábitat de Mammillaria eriacantha es consecuencia de la remoción de roca para utilizar el terreno con fines agrícolas, lo que ha provocado el aislamiento de las poblaciones de esta cactácea al quedar inmersas en una matriz de cultivos, por lo que 
su mecanismo de dispersión deberá ser tan eficiente como para evitar los cultivos (caña y chayote) que los rodean y poder colonizar otros sitios. En el caso específico del fragmento seis, se observó una estructura de tamaños uniforme, con un exceso de adultos y un déficit de plantas de menor tamaño. Una posible explicación es que este fragmento está rodeado por el pueblo de Trapiche del Rosario y al menos visualmente, está perturbado y contaminado de basura. $\mathrm{Pu}-$ diera ocurrir que los asentamientos humanos a su alrededor interrumpan la dispersión de semillas y la cubierta de basura impida la colonización, lo que podría promover la extinción local de $M$. eriacantha en ese fragmento.

El fuego también es un factor que reduce las poblaciones de Mammillaria eriacantha, ya que anualmente ocurre la quema de cañaverales para la zafra que eventualmente invaden los fragmentos donde no ha ocurrido la remoción de roca. Se ha demostrado que poblaciones de M. magnimamma que se encuentran en sitios sujetos al fuego, tienen una tasa de crecimiento poblacional menor a poblaciones que se encuentran en sitios no incendiados (Valverde et al., 2004). La estructura de tamaños de $M$. eriacantha en la mayoría de los fragmentos es similar a la estructura de tamaños general por lo que habría que estimar la pérdida anual de bosque tropical caducifolio por fuego y calcular con base en los datos de densidad de $M$. eriacantha, cuantos individuos se pierden anualmente.

Algunos trabajos han establecido que el tamaño mínimo para que una población sea viable es de 20 a 50 adultos o individuos reproductivos (Brussard, 1991; Burgman et al., 2001); pero esto varía dependiendo de cada especie. El tamaño mínimo viable deberá asegurar la supervivencia de la población a largo plazo y dependerá de eventos estocásticos y ambientales (Wilcox y Elderd, 2003). Consideramos que en este trabajo la población es suficientemente grande puesto que los adultos (individuos en floración) encontrados en una muestra de tan sólo 300 plantas representó el 18\% (altura > $9 \mathrm{~cm}$ ). Por otro lado, es poco probable que la explotación de plantas de Mammillaria eriacantha menores a $9 \mathrm{~cm}$ afecte el tamaño de la población, pero dado que M. eriacantha se distribuye homogéneamente al interior de los fragmentos, es alta la probabilidad de que plantas de $M$. eriacantha se pierdan como consecuencia del cambio de uso del suelo y de los incendios.

Se ha encontrado que Mammillaria eriacantha puede estar asociada a la rocosidad y a la cobertura de hierbas y leñosas (Castillo-Campos, 2003). Aquí, encontramos independencia entre estas características ambientales y las características poblaciones de $M$. eriacantha, lo cual corresponde con su distribución homogénea. Es probable que la diferencia en ambos estudios se deba a que aquí se relacionó la densidad y el valor de asimetría de tamaños y no a la probabilidad de presencia de $M$. eriacantha como ocurrió en el primer estudio (Castillo-Campos, 2003). Además las parcelas muestreadas en nuestro trabajo pudieran representar sólo una parte del hábitat, ya que Castillo-Campos (2003) muestreó un continuo de las coberturas (desde 0-100\%) y encontró que a mayor cobertura rocosa hay mayor probabilidad de presencia de $M$. eriacantha y a coberturas intermedias de hierbas y leñosas, también aumenta la probabilidad de encontrar a individuos de esta especie.

El alto grado de endemismo de las cactáceas, las convierten en una de las familias más vulnerables a modificaciones de su hábitat. Además, como son individuos de lento crecimiento, la probabilidad de recuperación también será lenta, particularmente si se extraen los adultos que son los productores de semillas. La información generada en este trabajo establece que al menos para el monitoreo y estudios poblacionales de Mammillaria eriacantha es suficiente muestrear dentro de pocos fragmentos de vegetación con una muestra pequeña (muestra $<10$ parcelas) dentro de ellos. No obstante que no se encontró relación entre las características ambientales del sitio de estudio con las características poblacionales de $M$. eriacantha, se recomienda evaluar otras características abióticas, como por ejemplo: la luz o características del suelo ( $\mathrm{pH}$, nutrientes, humedad). También sería importante que antes de emitir recomendaciones específicas sobre planes de conservación y/o manejo de esta especie se realicen estudios sobre su dinámica poblacional, en función de eventos como los incendios y la disminución de su hábitat para actividades agrícolas.

\section{Agradecimientos}

Agradecemos los comentarios sobre versiones anteriores del trabajo a: A. Montano, C. Montaña y M. Ferrer. Agradecemos a J. González-Astorga y J. Tolome por la facilitación del GPS y a Keith MacMillan por su colaboración con la versión en inglés del resumen. Con una versión preliminar de este trabajo S. Valencia-Díaz obtuvo el grado de especialista en Métodos Estadísticos en la Facultad de Estadística e Informática de la Universidad Veracruzana.

\section{Literatura citada}

Bárcenas-Luna R.T. 2003. Los cactos del Desierto Chihuahuense en México: Una evaluación del comercio, la administración y las prioridades de conservación. En: Robbins C.S. Ed. Comercio Espinoso: Comercio y Conservación de Cactos en el Desierto Chihuahuense, pp. II1-II65, TRAFFIC Norteamérica/Fondo Mundial para la Naturaleza, Washington DC.

Bell T., Freckleton R.P. y Lewis O.T. 2006. Plant pathogens drive density dependent seedling mortality in a tropical tree. Ecology Letters 9:569-574.

Bravo-Hollis H. y Sánchez-Mejorada H. 1991. Las Cactáceas de México. Vol. III. Universidad Autónoma de México, México, D.F.

Brussard P.F. 1991. The role of ecology in biological conservation. Ecological Applications 1:6-12.

Burgman M.A., Possingham H.P., Lynch A.J.J., Keith D.A., McCarthy M.A., Hopper S.D., Drury W.L., Passioura J.A. y Devries R.J. 2001. A method for setting the size of plant conserva- 


\section{Susana Valencia-Díaz ET AL.}

tion target areas. Conservation Biology 15:603-616.

Castillo-Campos G. 2003. Biodiversidad de la selva baja caducifolia en un sustrato rocoso de origen volcánico en el Centro del Estado de Veracruz, México. Tesis de Doctorado Universidad Autónoma Metropolitana. México, D.F. 203 pp.

Castillo-Campos G., Halffter G. y Moreno C.E. 2008. Primary and secondary vegetation patches as contributors to floristic diversity in a tropical deciduous forest landscape. Biodiversity Conservation 17:1701-1714.

Clark P.J. y Evans F.C. 1954. Distance to nearest neighbor as a measure of spatial relationships in population. Ecology 35:445-453.

Drezner T.D. y Lazarous B.L. 2008. The population dynamics of columnar and other cacti: A review. Geography Compass 2:1-29.

Flores-Palacios A. y Valencia-Díaz S. 2007. Local illegal trade reveals unknown diversity and involves a high species richness of wild vascular epiphytes. Biological Conservation 136:372-387.

Frankham R. 1998. Inbreeding and extinction: Island populations. Conservation Biology 12:665-675.

García E. 1988. Modificaciones al Sistema de Clasificación Climática de Köppen (para Adaptarlo a las Condiciones de la República Mexicana). Offset Larios, México, D.F.

Gilbert G.S. 2002. Evolutionary ecology of plant diseases in natural ecosystems. Annual Review of Phytopathology 40:13-43.

Godínez-Álvarez H., Valverde T. y Ortega-Baes P. 2003. Demographic trends in the Cactaceae. The Botanical Review 16:173-203.

Guevara-S S., Laborde-D J. y Sánchez-Ríos G. 2004. La fragmentación. En: Guevara-S S., Laborde-D J. y Sánchez-Ríos G. Eds. Los Tuxtlas. El Paisaje de la Sierra, pp. 111-134, Instituto de Ecología A.C./Unión Europea, Xalapa.

Haberman S.J. 1973. The analysis of residual in cross-classified tables. Biometrics 29:205-220.

Hanski I. 1998. Metapopulation dynamics. Nature 396:41-49.

Harrison S. 1991. Local extinction in a metapopulation context: an empirical evaluation. Biological Journal of the Linnean Society 42:73-88.

Hernández H.M. y Godínez-A H. 1994. Contribución al conocimiento de las cactáceas mexicanas amenazadas. Acta Botanica Mexicana 26:36-52.

Hou M-F, López-Pujol J., Quin H-N, Wang L-S, Liu Y. 2010. Distribution pattern and conservation priorities for vascular plants in Southern China: Guangxi Province a case of study. Botanical Studies 51:377-386.

Hunt D. 1992. CITES Cactaceae Checklist. Royal Botanical Gardens, Kew.

Jacquemyn H., Butaye J. y Hermy M. 2003. Influence of environmental and spatial variables on regional distribution of forest plant species in fragmented and changing landscape. Ecography 26:768-776.

Karron J.D. 1997. Genetic consequences of different patterns of distribution and abundance. En: Kuning W.E. y Gaston K.J. Eds. The Biology of Rarity Causes and Consequences of RareCommon Differences, pp 174-189, Springer, Ascot.

Keith D.A. 2000. Sampling designs, field techniques and analytical methods for systematic plant population surveys. Ecological Management and Restoration 1:125-139.
Larrea-Alcázar D., López R.P. y Barrientos D. 2005. The nurseplant effect of Prosopis flexulosa D.C. (Leg-Mim) in a dry valley of the Bolivian Andes. Ecotropicos 18:89-95.

Menges E.S. y Dolan R.W. 1998. Demographic viability of populations of Silene regia in Midwestern prairies: relationships with fire management, genetic variation, geographic location, population size and isolation. Journal of Ecology 86:63-78.

Morgan J.W. 1999. Effects of population size on seed production and germinability in an endangered, fragmented, grassland plant. Conservation Biology 13:266-273

Mueller-Dombois D. y Ellenberg H. 1974. Aims and Methods of Vegetation Ecology. John Wiley and Sons, Nueva York.

Reed D.H. 2004. Relationship between population size and fitness. Conservation Biology 19:563-568.

Schaeffer L.R., Mendenhall W. y Ott R.L. 1986. Elementos de Muestreo. Grupo Editorial Iberoamericana, México, D.F.

Seidler T.G. y Plotkin J.B. 2006. Seed dispersal and spatial pattern in tropical trees. PLoS Biology 4:2132-2137.

SEMARNAT [Secretaría del Medio Ambiente y Recursos Naturales]. 2010. Norma Oficial Mexicana NOM-059-SEMARNAT2010 Protección ambiental - Especies nativas de México de flora y fauna silvestres - Categorías de riesgo y especificaciones para su inclusión, exclusión o cambio.- Lista de especies en riesgo. Diario Oficial de la Federación 2a Sección, 30 de diciembre de 2010.

Sih A., Jonsson B.G. y Luikart G. 2000. Habitat loss: ecological, evolutionary and genetic consequences. Trends in Ecology and Evolution 15:132-134.

Silvertown J.W. y Lovett-Doust J. 1993. Introduction to Plant Population Biology. Blackwell Scientific Publications, Londres.

Valiente-Banuet A., Bolongaro-Crevenna A., Briones O., Ezcurra E., Rosas M., Núñez H., Barnard G. y Vázquez E. 1991. Spatial relationships between cacti and nurse shrubs in a semi-arid environment in central Mexico. Journal of Vegetation Science 2:15-20.

Valverde T., Quijas S., López-Villavicencio M. y Castillo S. 2004. Population dynamics of Mammillaria magnimamma Haworth. (Cactaceae) in a lava field in central Mexico. Plant Ecology 170:167-184.

Vergeer P., Rengelink R., Copal A. y Ouborg N.J. 2003. The interacting effects of genetic variation habitat quality and population size on performance of Succisa pratensis. Journal of Eco$\log y$ 91:18-26.

Vovides A.P., Luna V. y Medina G. 1997. Relación de algunas plantas y hongos mexicanos raros, amenazados o en peligro de extinción y sugerencias para su conservación. Acta Botanica Mexicana 39:1-42.

Wilcove D.S., Mc Lellan C.H. y Dobson A.P. 1986. Habitat fragmentation in the temperate zone. En: Soulé M.E. Ed. Conservation Biology. The Science of Scarcity and Diversity, pp. 237256, Sinauer, Sunderland.

Wilcox C. y Elderd B. 2003. The effect of density-dependent catastrophes on population persistence time. Journal of Applied Ecology 40: 859-871.

Zar J.H. 2010. Biostatistical Analysis. Prentice-Hall, Nueva Jersey.

Recibido: 24 de agosto de 2011

Aceptado: 16 de diciembre de 2011 